\title{
Complex Tracer Diffusion Dynamics in Polymer Solutions
}

\author{
Apostolos Vagias, ${ }^{1}$ Riccardo Raccis, ${ }^{2}$ Kaloian Koynov, ${ }^{1}$ Ulrich Jonas, ${ }^{3}$ Hans-Jürgen Butt, ${ }^{1}$ George Fytas,,${ }^{1,4 * *}$ \\ Peter Košovan, ${ }^{5,6, \dagger}$ Olaf Lenz, ${ }^{5}$ and Christian Holm ${ }^{5}$ \\ ${ }^{1}$ Max Planck Institute for Polymer Research, Ackermannweg 10, 55128 Mainz, Germany \\ ${ }^{2}$ Institute for Inorganic Chemistry, University of Köln, Greinstraße 6, 50939 Köln, Germany \\ ${ }^{3}$ Macromolecular Chemistry, University of Siegen, Adolf-Reichwein-Strasse 2, 57076 Siegen, Germany \\ ${ }^{4}$ Department of Materials Science, University of Crete and FORTH/ISEL, P.O. Box 1527, 71110 Heraklion, Greece \\ ${ }_{5}^{5}$ Institute for Computational Physics, Universität Stuttgart, Allmandring 3, 70569 Stuttgart, Germany \\ ${ }^{6}$ Department of Physical and Macromolecular Chemistry, Faculty of Science, Charles University in Prague, \\ Hlavova 8, 12000 Praha 2, Czech Republic
}

(Received 24 January 2013; published 21 August 2013)

\begin{abstract}
We employ fluorescence correlation spectroscopy (FCS) and coarse-grained molecular dynamics simulations to study the mobility of tracers in polymer solutions. Excluded volume interactions result in crowding-induced slowdown, depending only on the polymer concentration. With specific tracerpolymer attractions, the tracer is slowed down at much lower concentrations, and a second diffusion component appears that is sensitive to the polymer chain length. The two components can be resolved by FCS, only if the distance traveled by the tracer in the polymer-bound state is greater than the FCS focal spot size. The tracer dynamics can be used as a sensitive probe of the nature and strength of interactions, which—despite their local character-emphasize the role of chain connectivity.
\end{abstract}

Fluorescence correlation spectroscopy (FCS) is a technique for studying the dynamics of small molecules in complex environments [1-4]. It has become overwhelmingly popular in biological sciences due to its single-molecule sensitivity and noninvasive nature [5,6]. Fluctuations of the fluorescence intensity due to individual tracer molecules crossing the focal spot of the excitation laser beam reveal tracer dynamics [4,7]. Fitting the fluorescence intensity autocorrelation function $G(t)$ by a single Fickian process, one can extract the diffusion constant [4]. If $G(t)$ cannot be described by one-component diffusion, the interpretation becomes unclear unless the applied model can be validated independently $[2,8,9]$. This ambiguity limits the application of FCS and the amount of useful information obtainable on diffusion in the presence of interactions with the host environment. However, investigations of such media are of paramount importance for biomedical applications [10].

In this Letter, we systematically study the diffusion of several tracers that exhibit different interactions with PNiPAAm ( $N$-isopropyl acrylamide) terpolymers (see the Supplemental Material [11]). PNiPAAm is a thermoresponsive polymer utilized in biosensor applications [12,13]. We have deliberately chosen this simplified polymeric model system to mimic a crowded environment with possible attractive interactions frequently encountered in biological and soft matter systems [14-16]. In addition,

Published by the American Physical Society under the terms of the Creative Commons Attribution 3.0 License. Further distribution of this work must maintain attribution to the author(s) and the published article's title, journal citation, and DOI. we performed molecular dynamics (MD) simulations of a system that entails an interacting spherical tracer in a solution of bead-spring polymers. We compare simulated and measured $G(t)$ and demonstrate that in the case of attractive tracer-polymer interactions, a two-component diffusion process can model the experimental $G(t)$, whereas a comparable good fit of an anomalous diffusion model to $G(t)$ can be ruled out. The fast component corresponds to free diffusion, whereas the slow one stems from the polymer-bound tracer. We support this picture by constructing a simple binding model that explains the MD data in terms of fractions of bound and free tracers. The ability to resolve the two diffusion processes depends crucially on the FCS focal volume which defines the available length and time scales necessary to resolve the underlying processes $[17,18]$.

If not stated otherwise, our experiments were performed at $25^{\circ} \mathrm{C}$ in aqueous solutions of PNiPAAm terpolymers with $M_{w}=280 \mathrm{~kg} / \mathrm{mol}$ (hydrodynamic radius $R_{H} \approx$ $15 \mathrm{~nm}$ ) [19]. Four tracers were selected, on the account of different interactions with PNiPAAm: Alexa 647 and 488 $\left(\mathrm{A} 647, R_{H}=0.67 \mathrm{~nm} ; \mathrm{A} 488, R_{H}=0.54 \mathrm{~nm}\right)$, Rhodamine 6G (Rh6G, $\left.R_{H}=0.60 \mathrm{~nm}\right)$, and $\mathrm{CdSe} / \mathrm{ZnS}$ quantum dots (QD, $\left.R_{H}=6.1 \mathrm{~nm}\right)$. As compared to bulk solution, (i) A488 and A647 were depleted within a PNiPAAm hydrogel film at $25^{\circ} \mathrm{C}$, which indicates dominating repulsive interaction with the polymer; (ii) the QDs had a virtually constant concentration profile and Rh6G accumulated in the gel, suggesting stronger attractive interactions of Rh6G (see Fig. 1 of the Supplemental Material [11]). The FCS experiments were performed on a Confocor 2 FCS setup from Carl Zeiss with a $40 \times$ Plan Neofluar objective 
with numerical aperture NA $=1.2$ and water as the immersion fluid.

To investigate the effect of polymer-tracer interactions, we calculated $G(t)$ from MD simulations of generic tracer and polymer models. We used the standard Kremer-Grest model [20] with the purely repulsive (athermal) [21] WeeksChandler-Andersen potential [22] to account for the excluded volume of all particles, with a diameter of $\sigma$. This is approximately the size of four PNiPAAm monomers or the molecular tracers. Attractive polymer-tracer interactions were modeled by the Lennard-Jones (LJ) potential with an adjustable attraction parameter $\epsilon$. Electrostatic interactions were neglected, which was justified experimentally by the indifference to addition of salt (see Fig. 2 of the Supplemental Material [11]). Hence, the Rh6G-PNiPAAm attractive interactions are predominantly hydrophobic. Our model is not expected to quantitatively reproduce the experiment but should capture the essential physics.

Simulations were performed with 20 polymers consisting of 50 segments $\left(R_{H} \approx 25 \mathrm{~nm}\right), 20$ athermal and 5 attractive tracers per simulation box, in an implicit solvent employing a Langevin thermostat [20], using the ESPRESSO software [23,24]. To compute $G(t)$ from simulation trajectories, we used the formula [25]

$$
G(t)=\left\langle\exp \left(-\frac{\Delta x^{2}(t)}{w^{2}}-\frac{\Delta y^{2}(t)}{w^{2}}-\frac{\Delta z^{2}(t)}{s^{2} w^{2}}\right)\right\rangle_{t_{0}},
$$

where $\Delta x^{2}(t)=\left|x\left(t_{0}\right)-x\left(t_{0}+t\right)\right|^{2}, \Delta y^{2}$ and $\Delta z^{2}$ have analogous meanings, and $w$ and $s$ denote the focal volume dimensions. For convenience, we used $s=1$ and $w=30 \mathrm{~nm}$ for the intensity profile. In simulations, $w$ is not bound by the diffraction limit but by the ballistic motion on short time scales due to the Langevin thermostat [20]. The chosen $w$, yields $G(t)$ unaffected by the initial ballistic motion. It is about 10 times smaller than in the experiment, shifting the time scale of $G(t)$ by a factor of $10^{2}$.

The $G(t)$ curves for the mobility of A488 and Rh6G in dilute aqueous solutions of PNiPAAm at $25^{\circ} \mathrm{C}$ are shown in Fig. 1(a). In the absence of specific tracerpolymer interactions, $G(t)$ should be described by a single diffusion coefficient $[n=1$ in Eq. (2)]. This is indeed true for A488 and A647 (not shown) at almost all examined PNiPAAm concentrations, but not for Rh6G, as seen from the fits in Fig. 1(a). Instead, the equation [4]

$$
G(t)=\frac{Q(t)}{N} \sum_{i=1}^{n} F_{i}\left[\left(1+\frac{4 D_{i} t}{w^{2}}\right)^{-1}\left(1+\frac{4 D_{i} t}{s^{2} w^{2}}\right)^{-0.5}\right],
$$

where $n=2$ could adequately represent the experimental $G(t)$ [dashed line in Fig. 1(a)]. The fast and slow diffusion constants $D_{i}, i \in\{$ fast, slow $\}$, and the amplitudes $F_{i}$, $F_{\text {slow }}=\left(1-F_{\text {fast }}\right)$, are adjustable parameters, while $N$ denotes the number of diffusing fluorophores per focal volume. The initial decay function $Q(t)$ accounts for photophysical relaxation processes specific for a particular fluorophore, such as triplet decay or blinking (for the
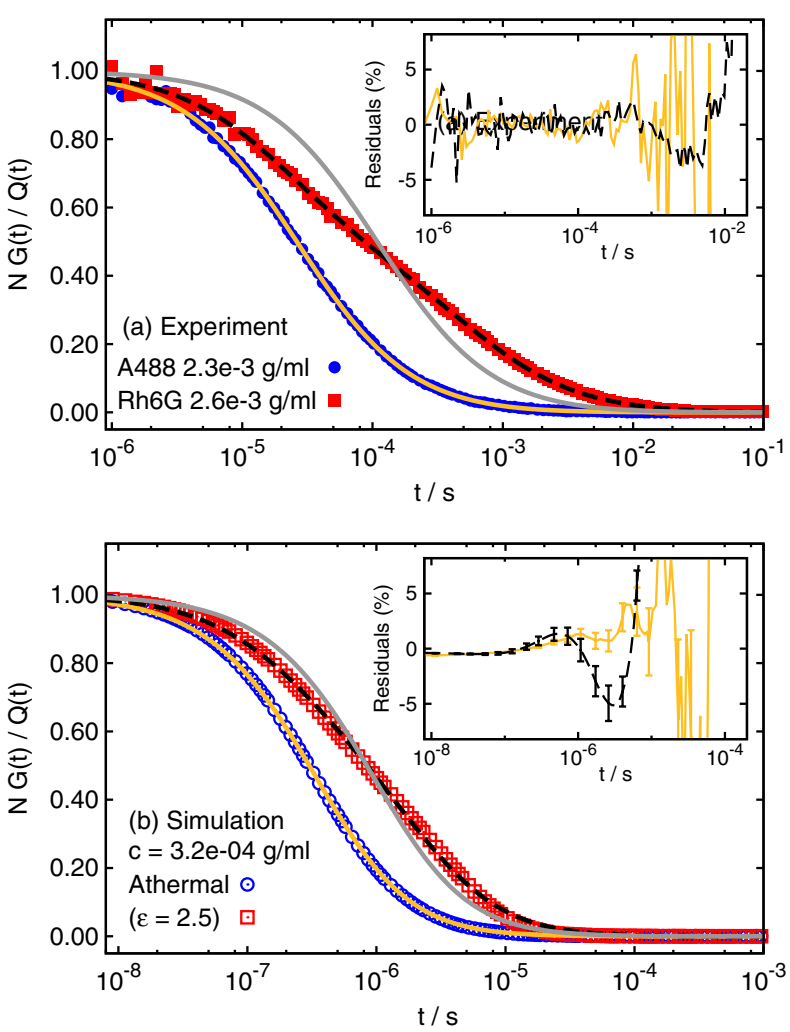

FIG. 1 (color online). Normalized FCS autocorrelation functions $G(t)$ from experiments and simulations: (a) Rh6G and A488 in dilute PNiPAAm solutions at $25^{\circ} \mathrm{C}$ and (b) the simulated athermal and attractive $\left(\epsilon=2.5 k_{B} T\right)$ tracers. Solid and dashed lines denote the single- and two-component representations of $G(t)$ by Eq. (2). Insets: residuals of the accepted fits (the colors match the corresponding fits).

QD). Fits to experimental and simulated $G(t)$ using a subdiffusive model that is often invoked in literature to describe complex shapes of $G(t)$ yielded a concentrationdependent exponent $0.1<\alpha(c)<1.0$. However, as we explain later, this model is inappropriate despite a comparable fit quality with the two-component model (see Fig. 4 of the Supplemental Material [11]).

Figure 1(b) shows $G(t)$ computed from the MD data. The observed single Fickian diffusion of A647 and A488 is well captured by the MD simulations of the athermal tracer. In agreement with the experiment, the MD simulations of the strongly attractive tracer $\left(\epsilon=2.5 k_{B} T\right)$, as the counterpart of Rh6G, yielded $G(t)$ that clearly deviates from single diffusion already at concentrations far below the overlap concentration $c^{*} \sim M / R_{H}^{3}$ [21]. The occurrence of two diffusive processes for $\mathrm{Rh} 6 \mathrm{G}$, however, is not due to a crowding effect solely determined by $c$, as reported for the single Fickian diffusion of noninteracting molecular tracers [26]. Instead, it is a polymeric effect, as indicated by the faster $G(t)$ decay of Rh6G in PNiPAAm with $M_{w}=90 \mathrm{~kg} / \mathrm{mol}$ at the same $c<c^{*}$ (see Fig. 2 of the Supplemental Material [11]). It is the connectivity of the polymer that alters the slow component of Rh6G 
dynamics, similar to a recent observation for Rh6G in poly (styrene sulfonate) solutions [27].

We further examined the slowdown relative to the free diffusivity $D / D_{0}$ as a function of $c$. For the athermal tracers displayed in Fig. 2(a), it has been experimentally shown that a superposition on a single master curve is successful in the plot of $D / D_{0}$ vs $c$ [26]. The fast component of Rh6G diffusion and of the simulated strongly attractive tracer $\left(\epsilon=2.5 k_{B} T\right)$ also follow this master curve. Polymer self-diffusion, $D_{\text {pol }}$, from both simulation and experiment superimpose in the plot vs $c / c^{*}$ in Fig. 2(b) on a master curve for macromolecular diffusants [26]. As $c^{*}$ depends on polymer molecular weight, such a representation scales out the effect of chain length. Remarkably, the slow component of Rh6G diffusion as well as the slow component of the simulated strongly attractive tracer
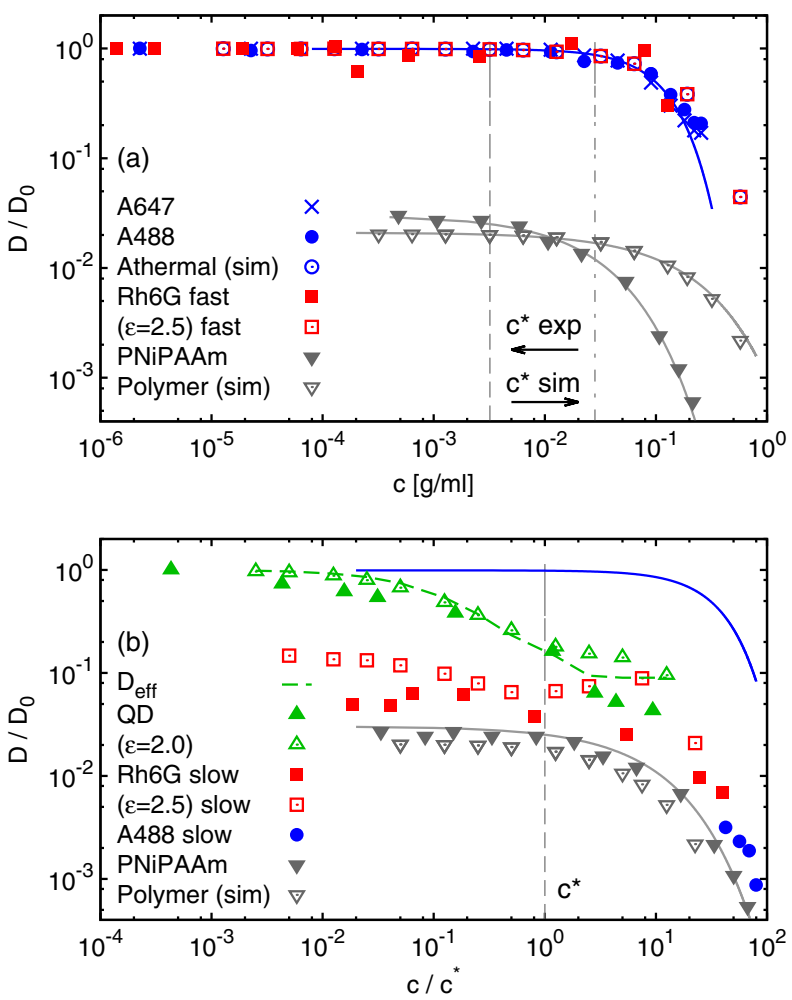

FIG. 2 (color online). Diffusion slowdown $D / D_{0}$ as a function of polymer concentration. (a) Crowding-induced tracer slowdown as a function of total polymer concentration $c$ : A647, A488, and the simulated athermal tracer (single diffusion) and the fast diffusion components of Rh6G and of the strongly attractive tracer $\left(\epsilon=2.5 k_{B} T\right)$. (b) Macromolecular tracer slowdown as a function of reduced concentration $c / c^{*}$ : polymer selfdiffusion and slow diffusion components of Rh6G and of the strongly attractive tracer. The diffusion of the QD and of the weakly attractive tracer $\left(\epsilon=2.0 k_{B} T\right)$ in (b) with its effective diffusion $D_{\text {eff }}$ described in the text follow neither type of master curve. The master curves for crowding-induced slowdown (blue) and the macromolecular tracer slowdown (gray) are shown as solid lines, together with data on polymer self-diffusion. Vertical lines indicate $c^{*}$. mimic the master curve for polymer self-diffusion. A slow component also shows up in the diffusion of A488 at high $c$, mimicking $D_{\text {pol }}$, similar to $D_{\text {slow }}$ of Rh6G and of the simulated strongly attractive tracer. This observation emphasizes the role of polymer chain length in the diffusion slowdown, when specific interactions are present.

For the weakly attractive QD, $G(t)$ is well represented by a single Fickian diffusion, but $D / D_{0}$ drops below unity even at $c \ll c^{*}$ [solid triangles in Fig. 2(b)]. Interestingly, the simulated attractive tracer with $\epsilon=2.0 k_{B} T$ [open triangles in Fig. 2(b)] also yields a single-component $G(t)$ with $D / D_{0}$ following the gradual decrease even at $c \ll c^{*}$. In view of this trend in both experiment and simulation, QD and the weakly attractive tracers exhibit intermediate behavior between the athermal (A647) and the strongly attractive (Rh6G) cases.

For Rh6G and the simulated attractive tracer, $F_{\text {slow }}$ was found to increase with PNiPAAm concentration (Fig. 3). The simulations also allow us to calculate the fraction of bound tracers $f$ by counting their number within the interaction range of the polymer. $F_{\text {slow }}$ from the simulated $G(t)$ and $f$ agree remarkably, indicating that $D_{\text {slow }}$ can indeed be attributed to the polymer-bound tracers. The $c$ dependence of $f$ and $F_{\text {slow }}$ can be represented by a simple binding model (dashed lines in Fig. 3) with binding constant $K=[\mathrm{T}][\mathrm{P}] /[\mathrm{TP}]$, where $[\mathrm{T}],[\mathrm{P}]$, and $[\mathrm{TP}]$ are the concentrations of free tracer, free polymer, and tracer-polymer complex. This also applies to $f$ of the weakly attractive tracer for which $F_{\text {slow }}$ is not available, since $G(t)$ is described by a single diffusion process. The experimental $F_{\text {slow }}$ of Rh6G in Fig. 3 increases with $c$ qualitatively similar to the simulations. However, a variation of $K$, which is the only adjustable parameter of the above model, can only shift the curve left or right but cannot account for the milder slope of the experimental $F_{\text {slow }}$.

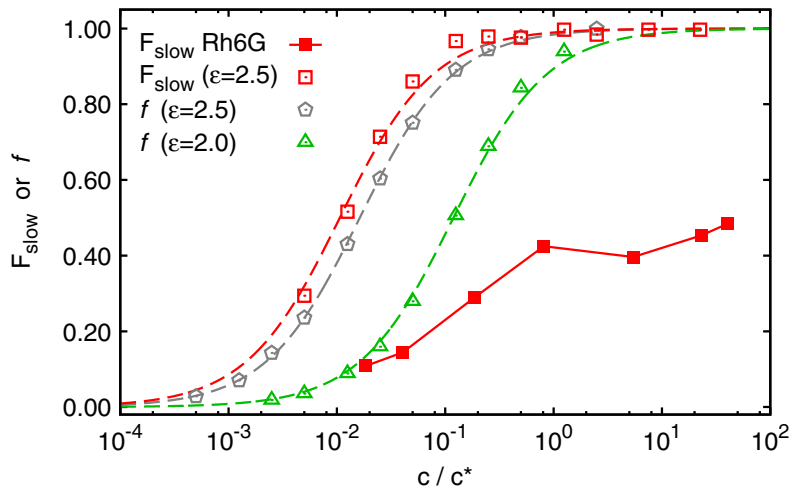

FIG. 3 (color online). The amplitude of the slow process $F_{\text {slow }}$ as a function of $c / c^{*}$. Solid and empty symbols refer to $F_{\text {slow }}$ from the experimental and simulated $G(t)$, respectively. The bound fraction $f$ obtained from the simulations for weakly and strongly attractive tracers is shown for comparison. Dashed lines are fits of the simple binding model with $K=94,61$, and 8.4 (from left to right). 
Phenomenologically, the observation could be accounted for by using $K$ which decreases with $c$. This, however, cannot be captured by our model with constant $\epsilon$.

Nevertheless, there is agreement between the experimental and simulated $D_{\text {fast }}$ and $D_{\text {slow }}$, conforming to universal master curves (Fig. 2). Note that $K \sim \exp \left(-U / k_{B} T\right)$ [28] implies that the binding strength and hence $F_{\text {slow }}$ and $f$ are sensitive to the interaction potential $U$. In contrast, $D_{\text {fast }}(c)$ (free tracer diffusion) is independent of specific polymer-tracer interactions, while $D_{\text {slow }}(c)$ (the polymerbound diffusion) only exists in the presence of specific interactions but its value is virtually independent of their strength. We argue that the underlying diffusion process is indeed two component in both cases, showing that $D(c)$ obtained from $G(t)$ of the weakly attractive case can be reconstructed using the effective diffusion coefficient $D_{\text {eff }}(c)=D_{\text {slow }} f(c)+D_{\text {fast }}[1-f(c)] \quad$ [dashed line in Fig. 2(b)]. Here, we assumed the same $D_{\text {slow }}$ and $D_{\text {fast }}$ as for $\epsilon=2.5 k_{B} T$ but used $f(c)$ for $\epsilon=2.0 k_{B} T$.

It is intriguing that small variations in the attraction change $G(t)$ qualitatively, from single- to two-component diffusion. This can be rationalized by comparing $w$ to the system-relevant length scales. In a dilute polymer solution, there is a dynamic exchange between the polymer-bound and free states of the tracer. The tracer undergoes free diffusion in the free volume between separated coils with $D_{0}$ for a time $t_{\text {free }}$ and diffuses bound to the polymer with $D_{\text {bound }} \approx D_{\text {pol }}$ for a time $t_{\text {bound }}$. The corresponding displacements are $L_{\text {free }}^{2} \sim D_{0} t_{\text {free }}$ and $L_{\text {bound }}^{2} \sim D_{\text {bound }} t_{\text {bound }}$. As discussed in Ref. [17], a particular process can be resolved by FCS only when its characteristic length scale is greater than $w$. Thus, $w<L_{\text {bound }}$ results in a bimodal $G(t)$ whereas $w>L_{\text {bound }}$ results in an effective unimodal $G(t)$. From the MD data for $\epsilon=2.5 k_{B} T$, we found a bimodal $G(t)$ with $D_{\text {bound }} \approx 4.0 \times 10^{-11} \mathrm{~m}^{2} / \mathrm{s} \approx 0.09 D_{0}$ and $t_{\text {bound }} \approx 2.2 \mu \mathrm{s}$, leading to $L_{\text {bound }} \approx 54 \mathrm{~nm}>w=30 \mathrm{~nm}$ (see Fig. 3 in the Supplemental Material [11]). For $\epsilon=2.0 k_{B} T, G(t)$ conforms to a unimodal shape with $D_{\text {eff }} \ll D_{0}$ and $t_{\text {bound }} \approx 0.45 \mu \mathrm{s}$. Assuming the same $D_{\text {bound }}$, we obtained $L_{\text {bound }} \approx 11 \mathrm{~nm}<w$. In this case, the two distinct mechanisms exist but are averaged out before the tracer leaves the focal spot, yielding the effective $D_{\text {eff }}(c)$. Since $t_{\text {bound }} \sim \exp \left(-U / k_{B} T\right)$, a minor increase in the attraction significantly increases $t_{\text {bound }}$. To illustrate the link between $L_{\text {bound }}$ and $w$, we show in Fig. 4 a snapshot of the simulation trajectory for the strongly attractive tracer, compared to focal spots of different sizes. Regions with high density of spheres correspond to small displacements and hence slow (bound) diffusion, while regions with low sphere density correspond to large displacements and hence fast (free) diffusion. In Fig. 4(a), both $L_{\text {free }}$ and $L_{\text {bound }}$ exceed the focal spot size, while in Fig. 4(b), the focal spot is greater than both $L_{\text {free }}$ and $L_{\text {bound }}$. The commensurability of $w$ and the size $r$ of fixed confinement domains have been shown earlier to result in an effective
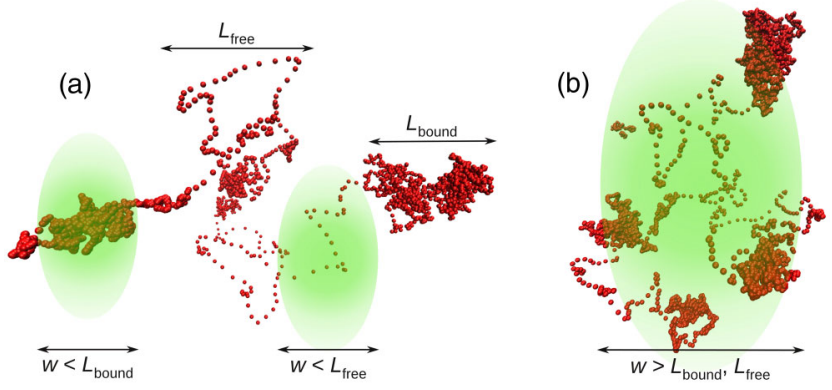

FIG. 4 (color online). Simulated trajectories of the attractive tracer $\left(\epsilon=2.5 k_{B} T\right)$ in dilute polymer solution mapped on focal spots (green) of different diameters $w$ : (a) $w \approx 30 \mathrm{~nm}<L_{\text {bound }}$, $L_{\text {free }}\left(\right.$ b) $w \approx 60 \mathrm{~nm}>L_{\text {bound }}, L_{\text {free }}$, where $L_{\text {bound }}\left(L_{\text {free }}\right)$ is the distance traveled by the bound (free) tracer. The spheres denote tracer positions separated by a constant time step. Regions with high density of spheres correspond to small displacements and slow (bound) diffusion while regions with low sphere density correspond to large displacements and fast (free) diffusion.

diffusion coefficient $[18,29]$. Despite an apparent similarity, what is commensurate with $w$ in our case is not the domain size $r \approx R_{H} \ll w$ but the length scale which arises from a combination of binding strength and domain mobility through $L_{\text {bound }}^{2} \sim D_{\text {bound }} t_{\text {bound }}$. Based on the analysis of length scales, we explain why subdiffusive model is not applicable in our case. Its use could be justified by $1 \mathrm{D}$ diffusion along the chain contour [30-32] or by an infinite hierarchy of binding traps $[33,34]$. The former is irrelevant because it could occur only below the resolution threshold since $R_{H} \ll w$. The latter is ruled out by the single exponential decay of the binding lifetimes (see Fig. 3 of the Supplemental Material [11]). Moreover, the simulated mean-square displacement (see Fig. 5 of the Supplemental Material [11]) exhibits no subdiffusion, rendering the apparent exponent $\alpha(c)$ a mere artifact.

In conclusion, probing tracer dynamics by means of FCS has shown that even in dilute polymer solutions, where the separation between polymers is much greater than their size, complex tracer diffusion can be observed. The measured $G(t)$ can be fitted by several different model equations by which no unique diffusion constant can be extracted and the exact underlying process cannot be revealed (Fickian vs anomalous diffusion). In the absence of analytical theory, MD simulations of a generic bead-spring polymer model and spherical tracers with no attraction (single diffusion), weak attraction (single slow diffusion), and strong attraction (double diffusion) to the polymer were shown to semiquantitatively capture the three experimentally observed behaviors. The two diffusion components could be attributed to bound and freely moving tracers. The lifetime of the bound state sensitively depends on the interaction energy, while the distance traveled by the bound tracer may or may not exceed the probed length scale. Consequently, FCS experiments yield quantitatively different decay functions, even though the microscopic diffusion mechanism does not 
change. Specific interactions of diffusants with the surrounding macromolecular environment are commonly encountered in soft matter and biology. Our results imply that they can be unambiguously identified from slowdown at high dilution. In this respect, this work lays the foundation for a systematic application of FCS to study single molecule transport in such environments.

The authors thank the DFG for support within the SPP 1259 "Intelligente Hydrogele" and Katja Nilles for the synthesis of PNiPAAm. P. K. acknowledges support from MSMT of the Czech Republic, Grant No. "LK 21302."

*fytas@mpip-mainz.mpg.de †kosovan@icp.uni-stuttgart.de

[1] W. Al-Soufi, B. Reija, M. Novo, S. Felekyan, R. Kühnemuth, and C. A. M. Seidel, J. Am. Chem. Soc. 127, 8775 (2005).

[2] J. Szymanski and M. Weiss, Phys. Rev. Lett. 103, 038102 (2009).

[3] B. Wang, S. M. Anthony, S. C. Bae, and S. Granick, Proc. Natl. Acad. Sci. U.S.A. 106, 15160 (2009).

[4] E. Elson, Biophys. J. 101, 2855 (2011).

[5] M. J. Saxton, Biophys. J. 103, 2411 (2012).

[6] S. A. Kim, K. G. Heinze, and P. Schwille, Nat. Methods 4, 963 (2007).

[7] D. Magde, W. W. Webb, and E. Elson, Phys. Rev. Lett. 29, 705 (1972).

[8] L. Kastrup, H. Blom, C. Eggeling, and S. W. Hell, Phys. Rev. Lett. 94, 178104 (2005).

[9] V. Ruprecht, S. Wieser, D. Marguet, and G. J. Schütz, Biophys. J. 100, 2839 (2011).

[10] J. Kim, J. Yoon, and R. C. Hayward, Nat. Mater. 9, 159 (2010).

[11] See Supplemental Material at http://link.aps.org/ supplemental/10.1103/PhysRevLett.111.088301 for further technical details of experimental sample preparation, fitting experimental $G(t)$, simulation model, and simulation protocol.

[12] C. J. Huang, J. Dostalek, and W. Knoll, Biosens. Bioelectron. 26, 1425 (2010).

[13] A. Mateescu, Y. Wang, J. Dostalek, and U. Jonas, Membranes 2, 40 (2012).
[14] D. Lumma, S. Keller, T. Vilgis, and J. O. Rädler, Phys. Rev. Lett. 90, 218301 (2003).

[15] A. Michelman-Ribeiro, D. Mazza, T. Rosales, T. J. Stasevich, H. Boukari, V. Rishi, C. Vinson, J.R. Knutson, and J. G. McNally, Biophys. J. 97, 337 (2009).

[16] S. Zustiak, R. Nossal, and D. Sackett, Biophys. J. 101, 255 (2011).

[17] J. Enderlein, Phys. Rev. Lett. 108, 108101 (2012).

[18] N. Destainville, A. Saulière, and L. Salomé, Biophys. J. 95, 3117 (2008).

[19] P. W. Beines, I. Klosterkamp, B. Menges, U. Jonas, and W. Knoll, Langmuir 23, 2231 (2007).

[20] G. S. Grest and K. Kremer, Phys. Rev. A 33, 3628 (1986).

[21] P. G. de Gennes, Scaling Concepts in Polymer Physics (Cornell University, Ithaca, New York, 1979).

[22] J. D. Weeks, D. Chandler, and H. C. Andersen, J. Chem. Phys. 54, 5237 (1971)

[23] A. Arnold, O. Lenz, S. Kesselheim, R. Weeber, F. Fahrenberger, D. Roehm, P. Košovan, and C. Holm, in Meshfree Methods for Partial Differential Equations VI, edited by M. Griebel and M. A. Schweitzer, Lecture Notes in Computational Science and Engineering Vol. 89 (Springer, New York, 2013), p. 1.

[24] H. J. Limbach, A. Arnold, B. A. Mann, and C. Holm, Comput. Phys. Commun. 174, 704 (2006).

[25] F. Höfling, K.-U. Bamberg, and T. Franosch, Soft Matter 7, 1358 (2011).

[26] T. Cherdhirankorn, A. Best, K. Koynov, K. Peneva, K. Müllen, and G. Fytas, J. Phys. Chem. B 113, 3355 (2009).

[27] P. Jia, Q. Yang, Y. Gong, and J. Zhao, J. Chem. Phys. 136, 084904 (2012).

[28] D. Chandler, Introduction to Modern Statistical Mechanics (Oxford University, Oxford, England, 1987).

[29] L. Wawrezinieck, H. Rigneault, D. Marguet, and P.-F. Lenne, Biophys. J. 89, 4029 (2005).

[30] O.G. Berg, R. B. Winter, and P.H. von Hippel, Biochemistry 20, 6929 (1981).

[31] M. Coppey, O. Bénichou, R. Voituriez, and M. Moreau, Biophys. J. 87, 1640 (2004).

[32] F. Tabatabaei, O. Lenz, and C. Holm, Colloid Polym. Sci. 289, 523 (2011).

[33] M. J. Saxton, Biophys. J. 92, 1178 (2007).

[34] N. Destainville, Soft Matter 4, 1288 (2008). 